\title{
Elevated red blood cell distribution width is associated with intrahepatic cholestasis of pregnancy
}

\author{
Zehra Vural Yilmaz ${ }^{1}$, Gulenay Gencosmanoglu Turkmen ${ }^{1}$, Korkut Daglar ${ }^{1}$, \\ Elif Yilmaz ${ }^{2}$, Ozgur Kara' ${ }^{1}$, Dilek Uygur ${ }^{1}$ \\ 'Zekai Tahir Burak Women's Health Care, Training and Research Hospital, Ankara, Turkey \\ ${ }^{2}$ Dr Sami Ulus Women's Health and Diseases Training and Research Hospital, Ankara, Turkey
}

\begin{abstract}
Objectives: Intrahepatic cholestasis of pregnancy is the most common pregnancy specific liver disease and related with adverse maternal and perinatal outcome. Red blood cell distribution width, an anisocytosis marker in a complete blood count, has been used as an inflammation marker in various diseases. However the association of red blood cell distribution width with intrahepatic cholestasis of pregnancy is unknown. We aimed to evaluate the relationship between red blood cell distribution width and intrahepatic cholestasis of pregnancy.

Material and methods: Ninety pregnant women with intrahepatic cholestasis of pregnancy and ninety healthy pregnant women were included in the study. Their clinical and laboratory characteristics including red blood cell distribution width, liver function tests, fasting and postprandial bile acid concentrations were analyzed.

Results: Serum red blood cell distribution width cell levels were significantly higher in pregnants with intrahepatic cholestasis of pregnancy than healthy pregnants. We also demonstrated that red blood cell distribution Width levels were higher in severe disease than mild disease and was significantly correlated with fasting and postprandial bile acid concentration in intrahepatic cholestasis of pregnancy group.

Conclusions: Our study showed that red blood cell distribution width, an easy and inexpensive marker; were associated with intrahepatic cholestasis of pregnancy and can be used as a diagnostic and prognostic marker in intrahepatic cholestasis of pregnancy.
\end{abstract}

Key words: red blood cell distribution width, intrahepatic cholestasis of pregnancy, severity

Ginekologia Polska 2017; 88, 2: 75-80

\section{INTRODUCTION}

Intrahepatic cholestasis of pregnancy (ICP) is the most common pregnancy specific liver disease and presents mainly in the third trimester with pruritis, elevated total serum bile acids and raised liver enzymes [1]. The incidence of the disease is reported between $0.2 \%$ and $2 \%$ depending on geographical location and ethnicity [2, 3]. ICP has been associated with serious and adverse pregnancy outcome such as preterm birth, nonreassuring fetal heart tracing, meconium staining of the amniotic fluid, and stillbirth [4].

The etiology of the ICP is complex and has not yet been understood. Multiple factors such as cholestatic effect of reproductive hormones in genetically susceptible pregnants, dietary and environmental factors play role in the pathogenesis of the disease $[5,6]$. The association between inflammatory processes and cholestatic liver disease has been reported in previous studies [5, 7]. Elevated concentrations of bile acids (BA) may lead to inflammatory response characterized by infiltration of inflammatory cells in hepatocytes and cause hepatocellular injury [5].

Red cell distribution width (RDW) is evaluated automatically during complete blood count (CBC) and shows variation of red blood cell volume. It is an anisocytosis marker and used especially for differential diagnosis of anemia [8]. However recent studies reported that systemic inflammation may lead to increase in RDW levels and it can be used as an inflammation marker in various diseases such as cardiovascular diseases (CVD), hypertension (HT), and 
thrombotic disorders [9]. Furthermore RDW is reported to be an independent risk factor for death in general population [10].

Although there are studies showing an association between RDW and many diseases associated with inflammation, there is no data about RDW levels and its relationship with ICP. Therefore we aimed to evaluate if there is an association between serum RDW levels and ICP and also with disease severity.

\section{MATERIAL AND METHODS}

This prospective case-control study was conducted at the Zekai Tahir Burak Women's Health Education and Research Hospital, a tertiary center in Ankara, Turkey. The Institutional Review Board of the hospital approved the study, and the universal principles of the Helsinki Declaration were applied. Informed consent was obtained from all participants.

Ninety pregnant women with diagnosis of ICP, 53 with mild disease and 27 with severe disease, during the period of January 2014 and January 2016 were included to the study. Ninety healthy pregnant women matched for maternal age in the third trimester within the same time were recruited as the control group. Pregnant with active infection, with chronic systemic disease (endocrinological, urogenital, cardiovascular, gastrointestinal, immunological, or oncological) and with multiple gestation were excluded from study. ICP was defined when the pregnant had unexplained pruritis without rashes in different parts of body with elevated serum TBA ( $\geq 10 \mathrm{mmol} / \mathrm{L}$ ) and/or raised liver enzymes. Pregnant women with ICP were divided into mild and severe group based on serum TBA levels of $10-40$ and $\geq 40 \mathrm{mmol} / \mathrm{L}$, respectively [1]. All the pregnant women with ICP were hospitalized and tested for $C B C$, liver function tests, hepatitis $A, B$ and $C$ virus for initial evaluation. All $C B C$ and the other blood analyses were conducted within two hours of blood sampling in the central laboratory of the hospital.

Demographic data, including maternal age, parity, gestational week, BMI, and the laboratory data of the routine $\mathrm{CBC}$ parameters in the first evaluation of the patient were recorded. Gestational age was determined based on the first day of the last menstrual period (LMP) and first trimester ultrasonographic measurement of the crown-rump length (CRL). If ultrasound dating differed from LMP dating by more than seven days, the estimated date was changed to ultrasound dating [11]. Gestational age at delivery, birth weight (BW), Neonatal Intensive Care Unit (NICU) admission, meconium staining of amniotic fluid and APGAR score at 5 minutes were recorded for assessment of perinatal outcome.

The data were analyzed using the Statistical Package for Social Sciences, Windows version 15.0 (SPSS, Chicago, IL,
USA). Descriptive statistics were expressed as mean \pm standard deviation and median (minimum-maximum) for numerical variables. Normality of the data distribution was assessed with the Kolmogorov-Smirnov test. Statistical significant differences between the groups were determined by Mann-Whitney $U$ test with Bonferroni correction. Categorical variables were expressed as number (percentage). Proportions were compared with Fisher's exact test or the chi-square test where appropriate. Associations between the variables were explored using the Pearson's correlation analyses. Logistic regression analyses were performed to calculate odds ratios (OR) with $95 \%$ confidence intervals. A p value $<0.05$ for were considered as statistically significant.

\section{RESULTS}

A total of 180 pregnant ( 90 with ICP and 90 without disease) were included to the study. There were no statistically significant differences between groups according to age, parity, body mass index (BMI) and gestational week. Mean hemoglobin and hematocrit concentration, white blood cell count (WBC) and platelet count were also similar between groups. Mean Alanine Aminotransferase (ALT) and Aspartate Aminotransferase (AST) levels were significantly higher in ICP group. Demographic data and laboratory parameters of ICP and control group are presented in Table 1.

There were also no significant differences in age, parity and BMI between mild and severe groups. Mean hemoglobin and hematocrit concentration, WBC and platelet count were also similar between mild disease and severe disease. Mean ALT level and mean fasting and postprandial

\begin{tabular}{|c|c|c|c|}
\hline Variables & $\begin{array}{l}\text { Control group } \\
\qquad(n=90)\end{array}$ & $\begin{array}{l}\text { ICP group } \\
(n=90)\end{array}$ & P value \\
\hline Age (years) & 26.86 .2 & $28.2 \pm 5.3$ & 0.103 \\
\hline GW at assessment & 34.93 .3 & $34.1 \pm 3.6$ & 0.121 \\
\hline Gravidity & $2(1-6)$ & $2(1-6)$ & 0.108 \\
\hline Parity & $1(0-4)$ & $0(0-4)$ & 0.051 \\
\hline BMI $\left[\mathrm{kg} / \mathrm{m}^{2}\right]$ & $24.3 \pm 1.2$ & $23.8 \pm 2.8$ & 0.19 \\
\hline ALT [U/L] & $16.5 \pm 16$ & $137 \pm 118$ & 0.0001 \\
\hline AST [U/L] & $19.6 \pm 8.8$ & $94.1 \pm 83.2$ & 0.0001 \\
\hline Hemoglobin [g/dL] & $11.5 \pm 1.06$ & $11.7 \pm 1.1$ & 0.168 \\
\hline Hemotocrit (\%) & $35.09 \pm 2.4$ & $35.9 \pm 3.3$ & 0.050 \\
\hline $\mathrm{WBC} \times 10^{3} \mathrm{~mL}$ & $9.7 \pm 2.1$ & $10.4 \pm 2.8$ & 0.058 \\
\hline Platelets $\times 10^{3} \mathrm{~mL}$ & $231.1 \pm 72.4$ & $245.1 \pm 64.2$ & 0.175 \\
\hline RDW (\%) & $14.2 \pm 1.7$ & $15.2 \pm 1.5$ & 0.0003 \\
\hline
\end{tabular}

Data expressed as mean \pm SD. GW - gestational week; BMI — body mass Index; ALT — alanine aminotransferase; AST — aspartate aminotransferase; WBC — white blood cell; RDW — red cell distribution width; ICP — intrahepatic cholestasis of pregnancy 
Table 2. Demographic and laboratory characteristics of mild and severe ICP group

\begin{tabular}{|c|c|c|c|}
\hline Variables & Mild group $(n=53)$ & Severe group $(n=37)$ & P value \\
\hline Age (years) & $28.1 \pm 4.8$ & $28.2 \pm 5.8$ & 0.981 \\
\hline GW at assessment & $34.9 \pm 2.3$ & $32.9 \pm 4.5$ & 0.019 \\
\hline Gravidity & $1(1-6)$ & $2(1-6)$ & 0.186 \\
\hline Parity & $0(0-4)$ & $0(0-2)$ & 0.503 \\
\hline $\mathrm{BMI}\left[\mathrm{kg} / \mathrm{m}^{2}\right]$ & $24.1 \pm 2.3$ & $24.4 \pm 1.8$ & 0.35 \\
\hline $\mathrm{ALT}[\mathrm{U} / \mathrm{L}]$ & $114.03 \pm 109.3$ & $169.4 \pm 125.2$ & 0.028 \\
\hline $\mathrm{AST}[\mathrm{U} / \mathrm{L}]$ & $81.7 \pm 82.2$ & $112 \pm 82.4$ & 0.089 \\
\hline Fasting TBA [mmol/L] & $21.3 \pm 8.7$ & $69.5 \pm 70.7$ & 0.0004 \\
\hline Postprandial TBA [mmol/L] & $21.5 \pm 12.7$ & $68.9 \pm 62$ & 0.0001 \\
\hline Hemoglobin $[\mathrm{g} / \mathrm{dL}]$ & $11.7 \pm 1.1$ & $11.8 \pm 1.2$ & 0.734 \\
\hline Hemotocrit (\%) & $35.8 \pm 3.0$ & $36.1 \pm 3.6$ & 0.678 \\
\hline $\mathrm{WBC} \times 10^{3} \mathrm{~mL}$ & $10.3 \pm 3.0$ & $10.4 \pm 2.7$ & 0.890 \\
\hline Platelets $\times 10^{3} \mathrm{~mL}$ & $245.4 \pm 63.8$ & $244.8 \pm 65.6$ & 0.967 \\
\hline RDW (\%) & $14.9 \pm 1.6$ & $15.8 \pm 1.1$ & 0.006 \\
\hline
\end{tabular}

Data expressed as mean \pm SD. GW — gestational week; BMI — body mass Index; ALT — alanine aminotransferase; AST — aspartate aminotransferase; TBA — total bile acid concentration; WBC — white blood cell; RDW — red cell distribution width; ICP — intrahepatic cholestasis of pregnancy

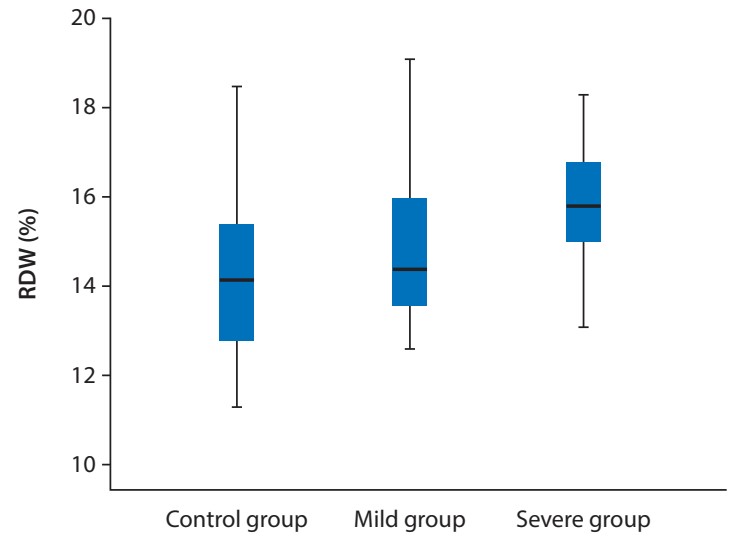

Figure 1. Comparison of RDW levels in the control, mild and severe ICP groups

total bile acid concentration (TBA) levels were significantly higher in severe group. Demographic data and laboratory parameters of mild and severe ICP groups are showed in Table 2.

In our study population mean RDW value was significantly higher in pregnants with ICP $(14.2 \pm 1.7)$ than without disease $(15.2 \pm 1.5)(p=0.003)$. There was also significant difference between pregnant with mild disease and severe disease $(14.9 \pm 1.6,15.8 \pm 1.1 ; p=0.006$ respectively) (Fig. 1). In correlation analyses in ICP group RDW was correlated with the fasting TBA ( $r=0.231, p=0.029)$ (Fig. 2) and postprandial TBA $(r=0.245, p=0.024)$ (Fig. 3).

RDW was also positively and significantly correlated with ALT levels $(r=0.231, p=0.001)$ and AST levels $(r=0.235$, $\mathrm{p}=0.001)$ in pregnant with ICP.

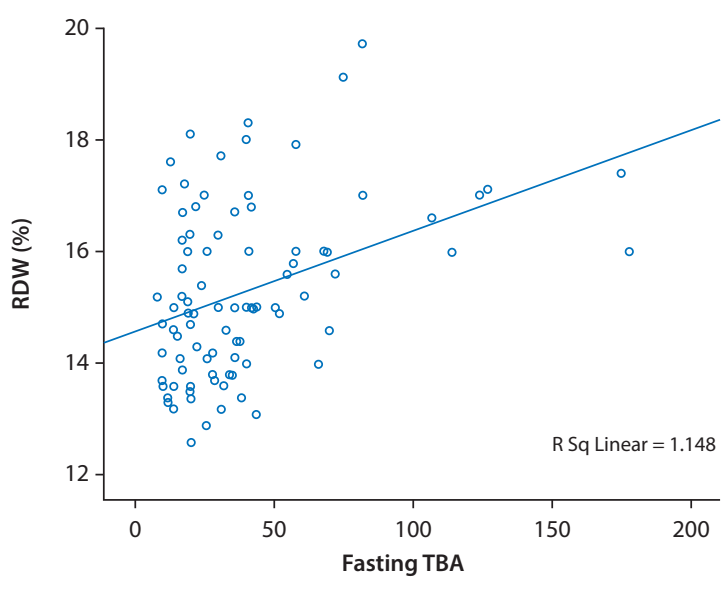

Figure 2. Correlation between RDW level and Fasting TBA $(r=0.231$, $p=0.029$ )

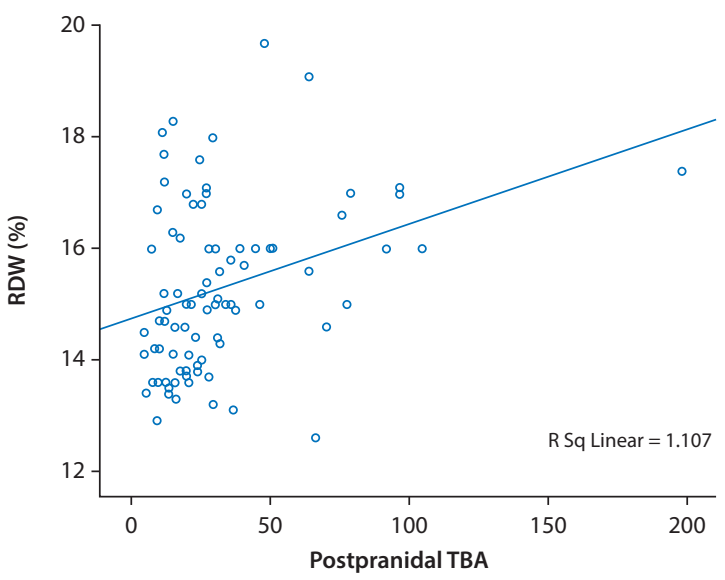

Figure 3. Correlation between RDW level and Postprandial TBA $(r=0.245, p=0.024)$ 
Table 3. The perinatal outcomes of control and ICP groups

\begin{tabular}{|l|c|c|c|}
\hline Variables & Control group $(\mathbf{n}=\mathbf{9 0})$ & ICP group $(\mathbf{n}=\mathbf{9 0})$ & P value \\
\hline GW at delivery & $38.1 \pm 1.4$ & $36.2 \pm 1.8$ & 0.0009 \\
\hline BW $[\mathrm{g}]$ & $3165 \pm 551$ & $2845 \pm 487$ & 0.0005 \\
\hline $\mathrm{BW}<10^{\text {th }}$ percentile, $\mathrm{n}(\%)$ & $3(3.3 \%)$ & $4(4.4 \%)$ & 0.7 \\
\hline Meconium staining of AF, $\mathrm{n}(\%)$ & $10(11.1 \%)$ & $12(13.3 \%)$ & 0.64 \\
\hline 5 minute APGAR $\leq 7, \mathrm{n}(\%)$ & $3(3.33 \%)$ & $10(11.1 \%)$ & 0.044 \\
\hline NICU admission, $\mathrm{n}(\%)$ & $4(4.4 \%)$ & $12(13.3 \%)$ & 0.036 \\
\hline
\end{tabular}

Data expressed as mean \pm SD, number(\%). GW - gestational week; BW — birth weight; AF — amniotic fluid; NICU — Neonatal Intensive Care Unit

Table 4. RDW values with perinatal complications in pregnants with ICP

\begin{tabular}{|l|c|c|}
\hline & RDW & P value \\
\hline $\mathrm{BW}<10^{\text {th }}$ percentile(+) & $14.7 \pm .9$ & 0.39 \\
\hline $\mathrm{BW}<10^{\text {th }}$ percentile(-) & $15.3 \pm 1.5$ & \\
\hline Meconium staining of AF(+) & $16.5 \pm 1.3$ & 0.024 \\
\hline 5 minute APGAR $\leq 7(+)$ & $15.08 \pm 1.4$ & \\
\hline 5 minute APGAR $\leq 7(-)$ & $16.1 \pm 1.8$ & 0.08 \\
\hline NICU admission (+) & $15.2 \pm 1.4$ & \\
\hline NICU admission (-) & $16.1 \pm 1.8$ & 0.08 \\
\hline
\end{tabular}

Data expressed as mean \pm SD. BW - birth weight; AF — amniotic fluid; NICU - Neonatal Intensive Care Unit

The perinatal outcomes of the pregnants with ICP and controls were presented in the Table 3. There were no cases of intrauterine fetal demise in any of the groups.BW and gestational age at birth were lower in the ICP group than the control group ( $p=0.0005$ and $p=0.0009$, respectively) (Tab. 3). In Pearson correlation analyses in pregnant women with ICP, there were no correlation between RDW and BW and gestational age at $\operatorname{birth}(r=-0.067, p=0.53 ; r=-0.068$, $\mathrm{p}=0.52$; respectively). In subgroup analyses in pregnants with ICP; mean RDW levels were significantly higher in pregnants with meconium staining than without meconium staining $(16.5 \pm 1.3,15.08 \pm 1.4 ; p=0.024$ respectively). However there were no differences in RDW values in terms of other perinatal complications in pregnants complicated with ICP (Tab. 4). In logistic regression analyses when considering serum RDW value $>15.7$ (\%), the risk of meconium staining increased 6.4 fold ( $95 \% \mathrm{Cl}: 1.5-24.1 ; \mathrm{p}=0.011)$.

\section{DISCUSSION}

In the present study we showed that RDW was significantly higher in pregnants with ICP than healthy pregnant controls. It was also significantly higher in severe disease than mild disease in subgroup analyses. We have reported positive and significant correlation between serum RDW level and fasting and postprandial total bile acid concentration and liver function tests in ICP patients. To the best of our knowledge this is the first study that evaluated and demonstrated an association between RDW level and ICP.

The exact pathogenesis of ICP is unknown but association between inflammation and disease has been previously reported in literature [7] .Elevated bile acid concentrations may lead to the release of several proinflammatory mediators and trigger inflammatory response in hepatocytes with infiltration of inflammatory cells into the liver [5, 12, 13]. The activated inflammatory cells cause significant liver injury [13] and liver's local intracellular response to inflammation determines the clinical outcome and prognosis of the disease [7]. However the mechanism how cholestasis stimulates the production of proinflammatory mediators in the liver has not been completely understood yet.

Recent studies demonstrated increased circulating levels of systemic and vascular inflammatory markers in pregnancies with ICP $[12,14,15]$. Systemic inflammation can be measured by a variety of serum markers and it is indicated that hematological indices and ratio of blood cell subtypes in CBC might have been used as an inflammatory marker in many diseases. RDW, a hematological parameter in $C B C$, shows red blood cell size and it is routinely used for differential diagnosis of anemia [16]. In recent studies, it is reported that systemic inflammation may cause an increase in RDW and it can be used as an alternative diagnostic inflammation and oxidative stress marker in many diseases such as CVD, renal diseases, thrombotic and hypertensive disorders $[9,16]$. Furthermore; $\mathrm{Hu} \mathrm{Z}$ et al. found increased RDW levels and showed RDW as a potential prognostic marker in different types of liver disease such as liver cirrhosis after HBV infection, primary hepatocellular carcinoma, and primary biliary cirrhosis [17].

Inflammation may impair iron metabolism and can cause release of inflammatory cytokines that inhibit red cell maturation and lead to immature erythrocytes to enter circulation. This may explain the association between increased RDW levels and inflammation [18, 19]. Elevated level of RWD has also been found to be associated with poor outcome and independent marker of mortality in these patients $[20,21]$. 
Systemic inflammation has been suggested to play a role in the etiology of certain obstetric complications such as preeclampsia, preterm delivery, and gestational diabetes $[22,23]$. Erdoğan et al found no association between RDW levels and gestational diabetes mellitus [24]. Kurt et al. found that RDW was associated with preeclampsia and with severity of the disease [25] .Otherwise Abdullahi et al could not find an association between serum RDW level and the presence of preeclampsia [26]. However, little is known and there is no study about if there is an association between RDW level and ICP. We found increased levels in pregnants complicated with ICP than healthy pregnants in our study.

ICP is associated with increased risk of preterm delivery, meconium passage, intrapartum fetal distress and stillbirth $[27,28]$. Due to the high rates of stillbirth and neonatal complications early delivery is recommended for these pregnants [29]. In literature there are many studies about the predictors of adverse neonatal outcome in these patients however optimal outcome predictors and timing of delivery are unknown. Serum TBA level $>40 \mathrm{mmol} / \mathrm{L}$ were found to be associated with increased risk of meconium staining, low APGAR scores and stillbirth in recent studies [30, 31]. We found lower BW and lower gestational age at birth in pregnants with ICP than healthy pregnants similar with the literature due to our policy of early delivery of these patients. Our findings also showed that high RDW levels were associated with the incidence of meconium staining in pregnants with ICP.

\section{CONCLUSIONS}

In conclusion, the present study demonstrated an association between that serum RDW level and ICP for the first time in literature. RDW, an easy, inexpensive and routinely reported parameter in a routinely performed CBC test might allow significant diagnostic and prognostic information in patients with ICP. However, further studies with large sample sizes are needed to understand the causative role of RDW in pregnants with ICP.

\section{Conflict of interest}

Authors declare no conflict of interest.

\section{REFERENCES}

1. Geenes V. Intrahepatic cholestasis of pregnancy. World Journal of Gastroenterology. 2009; 15(17): 2049-2066, doi: 10.3748/wjg.15.2049.

2. Lammert F, Marschall HU, Glantz A, et al. Intrahepatic cholestasis of pregnancy: molecular pathogenesis, diagnosis and management. J Hepatol. 2000; 33(6): 1012-1021, indexed in Pubmed: 11131439.

3. Reyes H, Báez ME, González MC, et al. Prevalence of intrahepatic cholestasis of pregnancy in Chile. Ann Intern Med. 1978; 88(4): 487-493, indexed in Pubmed: 637428.

4. Heinonen S, Kirkinen P. Pregnancy outcome with intrahepatic cholestasis. Obstet Gynecol. 1999; 94(2): 189-193, indexed in Pubmed: 10432125.

5. Allen $\mathrm{K}$, Jaeschke $\mathrm{H}$, Copple BL. Bile acids induce inflammatory genes in hepatocytes: a novel mechanism of inflammation during obstruc- tive cholestasis. Am J Pathol. 2011; 178(1): 175-186, doi: 10.1016/j. ajpath.2010.11.026, indexed in Pubmed: 21224055.

6. Woolbright $\mathrm{BL}$, Jaeschke $\mathrm{H}$. Novel insight into mechanisms of cholestatic liver injury. World J Gastroenterol. 2012; 18(36): 4985-4993, doi: 10.3748/wjg.v18.i36.4985, indexed in Pubmed: 23049206.

7. Kosters A, Karpen SJ. The role of inflammation in cholestasis: clinical and basic aspects. Semin Liver Dis. 2010; 30(2): 186-194, doi: 10.1055/s-00301253227, indexed in Pubmed: 20422500.

8. Romero Artaza J, Carbia CD, Ceballo MF, et al. Red cell distribution width (RDW): its use in the characterization of microcytic and hypochromic anemias. Medicina (B Aires). 1999; 59(1): 17-22, indexed in Pubmed: 10349113.

9. Montagnana M, Cervellin G, Meschi T, et al. The role of red blood cell distribution width in cardiovascular and thrombotic disorders. Clin Chem Lab Med. 2011; 50: 635-641.

10. Perlstein TS, Weuve J, Pfeffer MA, et al. Red blood cell distribution width and mortality risk in a community-based prospective cohort. Arch Intern Med. 2009; 169(6): 588-594, doi: 10.1001/archinternmed.2009.55, indexed in Pubmed: 19307522.

11. Committee opinion no 611: method for estimating due date. Obstet Gynecol. 2014; 124(4): 863-866, doi: 10.1097/01.AOG.0000454932.15177. be, indexed in Pubmed: 25244460.

12. O'Brien KM, Allen KM, Rockwell CE, et al. IL-17A synergistically enhances bile acid-induced inflammation during obstructive cholestasis. Am J Pathol. 2013; 183(5): 1498-1507, doi: 10.1016/j.ajpath.2013.07.019, indexed in Pubmed: 24012680.

13. Gujral JS, Farhood A, Bajt ML, et al. Neutrophils aggravate acute liver injury during obstructive cholestasis in bile duct-ligated mice. Hepatology. 2003; 38(2): 355-363, doi: 10.1053/jhep.2003.50341, indexed in Pubmed: 12883479.

14. Kirbas A, Biberoglu E, Ersoy AO, Dikmen AU, Koca C, Erdinç S et al. The role of interleukin-17 in intrahepatic cholestasis of pregnancy. J Matern Fetal Neonatal Med. 2015; 7: 1-5.

15. Biberoglu E, Kirbas A, Daglar K, et al. Role of inflammation in intrahepatic cholestasis of pregnancy. Journal of Obstetrics and Gynaecology Research. 2016; 42(3): 252-257, doi: 10.1111/jog.12902.

16. Salvagno GL, Sanchis-Gomar F, Picanza A, et al. Red blood cell distribution width: A simple parameter with multiple clinical applications. Crit Rev Clin Lab Sci. 2015; 52(2): 86-105, doi: 10.3109/10408363.2014.992064, indexed in Pubmed: 25535770.

17. Hu Z, Sun Yi, Wang Q, et al. Red blood cell distribution width is a potential prognostic index for liver disease. Clin Chem Lab Med. 2013; 51(7): 14031408, doi: 10.1515/cclm-2012-0704, indexed in Pubmed: 23314558.

18. Weiss G, Goodnough LT. Anemia of chronic disease. N Engl J Med. 2005 352(10): 1011-1023, doi: 10.1056/NEJMra041809, indexed in Pubmed: 15758012.

19. Pierce CN, Larson DF. Inflammatory cytokine inhibition of erythropoiesis in patients implanted with a mechanical circulatory assist device. Perfusion. 2005; 20(2): 83-90, indexed in Pubmed: 15918445.

20. Zalawadiya SK, Veeranna V, Niraj A, et al. Red cell distribution width and risk of coronary heart disease events. Am J Cardiol. 2010; 106(7): 988-993, doi: 10.1016/j.amjcard.2010.06.006, indexed in Pubmed: 20854962.

21. Patel KV, Semba RD, Ferrucci L, et al. Red blood cell distribution width and the risk of death in middle-aged and older adults. Arch Intern Med. 2009; 169(5):515-523, doi: 10.1001/archinternmed.2009.11, indexed in Pubmed: 19273783.

22. Borzychowski AM, Sargent IL, Redman CWG. Inflammation and pre-eclampsia. Semin Fetal Neonatal Med. 2006; 11(5): 309-316, doi: 10.1016/j. siny.2006.04.001, indexed in Pubmed: 16828580.

23. Romero R, Espinoza J, Gonçalves LF, et al. Inflammation in preterm and term labour and delivery. Semin Fetal Neonatal Med. 2006; 11(5): 317-326, doi: 10.1016/j.siny.2006.05.001, indexed in Pubmed: 16839830.

24. Erdoğan S, Ozdemir O, Doğan HO, et al. Liver enzymes, mean platelet volume, and red cell distribution width in gestational diabetes. Turk J Med Sci. 2014; 44(1): 121-125.

25. Kurt RK, Aras Z, Silfeler DB, et al. Relationship of red cell distribution width with the presence and severity of preeclampsia. Clin ApplThromb Hemost. 2015 Mar;21(2):128-31 doi: 10 1177/1076029613490827 Epub.; 2013: May, doi: $10.1177 / 1076029613490827$.

26. Abdullahi $\mathrm{H}$, Osman A, Rayis DA, et al. Red blood cell distribution width is not correlated with preeclampsia among pregnant Sudanese women. Diagn Pathol. 2014; 9: 29, doi: 10.1186/1746-1596-9-29, indexed in Pubmed: 24499498.

27. Arrese $\mathrm{M}$, Reyes $\mathrm{H}$. Intrahepatic cholestasis of pregnancy: a past and present riddle. Ann Hepatol. 2006; 5(3): 202-205, indexed in Pubmed: 17060884. 
28. Rook M, Vargas J, Caughey A, et al. Fetal outcomes in pregnancies complicated by intrahepatic cholestasis of pregnancy in a Northern California cohort. PLoS One. 2012; 7(3): e28343, doi: 10.1371/journal. pone.0028343, indexed in Pubmed: 22403605

29. Lo J, Shaffer B, Allen A, et al. Intrahepatic cholestasis of pregnancy and timing of delivery. The Journal of Maternal-Fetal \& Neonatal Medicine. 2014; 28(18): 2254-2258, doi: 10.3109/14767058.2014.984605.
30. Glantz A, Marschall HU, Mattsson LA. Intrahepatic cholestasis of pregnancy: Relationships between bile acid levels and fetal complication rates. Hepatology. 2004; 40(2): 467-474, doi: 10.1002/hep.20336, indexed in Pubmed: 15368452

31. Geenes V, Chappell LC, Seed PT, et al. Association of severe intrahepatic cholestasis of pregnancy with adverse pregnancy outcomes: a prospective population-based case-control study. Hepatology. 2014; 59(4): 1482-1491, doi: 10.1002/hep.26617, indexed in Pubmed: 23857305. 